\title{
Ischaemic heart disease in young hypertensive women
}

Sir,

The authors of this paper (Br Heart $\mathcal{F} 1980$; 43: 80-7) do not appear to differentiate between ST and $T$ wave abnormalities confined to the lateral leads and those found in other leads. It is therefore possible that in some of their cases the repolarisation abnormalities which they observed may have been a result of left ventricular hypertrophy rather than of ischaemic heart disease.

It is not sufficiently recognised that $S T$ and $T$ wave abnormalities resembling those of myocardial ischaemia and without increased $R$ and $S$ wave voltage may be found in patients with "pure" left ventricular hypertrophy, that is patients who give no history of angina and show no abnormality on coronary arteriography.

\section{David Short, Cardiac Department, Aberdeen Royal Infirmary, Foresterhill, Aberdeen AB9 2ZB.}

This letter was shown to Dr Mackay and his coauthors who reply as follows.

Sir,

Dr Short is correct in expressing concern about the specificity of electrocardiographic findings supposed to indicate myocardial ischaemia; we share some of his concern. We agree that ST segment and $T$ wave changes occurring in left ventricular hypertrophy and strain are difficult to distinguish from ST and $T$ wave changes occurring, in the same leads sometimes, in ischaemia without left ventricular hypertrophy. Evidence that the former is not ischaemic is scanty. We also recognise, and discuss in our paper, the occurrence of ST and T changes without evidence of coronary disease, and the occurrence of coronary disease without ST and $T$ wave changes.

Our main case rests, not with ST segment and $T$ wave changes on the electrocardiogram, but with angina pectoris and the clinical manifestations of ischaemic heart disease; 22 per cent of the young women in our series of patients with diastolic blood pressures of $120 \mathrm{mmHg}$ or more had a clear history of angina pectoris.
A Mackay, A M M Cumming, J J Brown, A F Lever, J I S Robertson, MRC Blood Pressure Unit, Western Infirmary, Glasgow G11 6NT. 